\title{
Spatial resolution of unmanned aerial vehicles acquired imagery as a result of different processing conditions
}

\author{
Jaroslav Kubišta ${ }^{1,2 *}$, Peter Surový ${ }^{1}$ \\ ${ }^{1}$ Czech University of Life Sciences, Faculty of Forestry and Wood Sciences, Prague, Kamýcká 129, \\ CZ-165 00 Praha, Czech Republic \\ ${ }^{2}$ Forest Management Institute, Nábřežní 1326, CZ-250 01 Brandýs nad Labem, Czech Republic
}

\begin{abstract}
Increasing availability of Unmanned aerial vehicles (UAV) and different software for processing of UAV imagery data brings new possibilities for on-demand monitoring of environment, making it accessible to broader spectra of professionals with variable expertise in image processing and analysis. This brings also new questions related to imagery quality standards. One of important characteristics of imagery is its spatial resolution as it directly impacts the results of object recognition and further imagery processing. This study aims at identifying relationship between spatial resolution of UAV acquired imagery and variables of imagery acquiring conditions, especially UAV flight height, flight speed and lighting conditions. All of these characteristics has been proved as significantly influencing spatial resolution quality and all subsequent data based on this imagery. Higher flight height as well as flight speed brings lower spatial resolution, whereas better lighting conditions lead to better spatial resolution of imagery. In this article we conducted a study testing various heights, flight speeds and light conditions and tested the impact of these parameters on Ground Resolved Distance (GRD). We proved that from among the variables, height is the most significant factor, second position is speed and finally the light condition. All of these factors could be relevant for instance in implementation of $\mathrm{UAV}$ in forestry sector, where imagery data must be often collected in diverse terrain conditions and/or complex stand (especially vertical) structure, as well as different weather conditions.
\end{abstract}

Key words: spatial resolution; ground resolved distance; light conditions; object identification; forestry sector

Editor: Bohdan Konôpka

\section{Introduction}

Unmanned aerial vehicles (UAV) or Unmanned aerial systems (UAS) have recently become increasingly available and thus ceased to be the prerogative of the army, and few scientific institutes. At present, inexpensive, user-friendly UAVs, with sufficient potential for aerial imaging can be easily purchased. Together with recent development in processing of digital imagery this brings new opportunities for on-demand monitoring available even for non-specialized practitioners (Pricope et al. 2019).

Consequently, new questions related to UAV imagery do emerge, such as what is the smallest object that can be identified on the image acquired on certain flight height, or what are the minimum required lighting conditions to perform imagery obtaining mission suitable for measurement of selected forest or tree parameters.

In other words, UAV imagery is facing new challenges in terms of describing requirements necessary to meet expected quality (Lee \& Sung, 2016) or quality standardization (Meißner et al. 2018). These challenges would be managed with regard to specific natural conditions. That is very relevant in forestry, a sector which is typical with very complex and variable conditions as for topography and forest stand traits. Spatial resolution is one of the basic characteristics of digital imagery with significant impact on object recognition results, therefore influencing any output derived from original UAV imagery. The smallest detectable object is related to this resolution and for the user interested in mensuration of particular variable or object (crown, individual tree), the information about the spatial resolution is crucial. Spatial resolution is often described by Ground Sampling Distance (GSD) (Orych 2015) which is a measure, that builds on known geometric parameters of camera and distance between camera and target.

Few studies examine further concepts such as Resolution, Resolution power (Lee \& Sung 2016), Ground resolved distance (GRD) (Orych 2015) or True Ground 
sample distance (tGSD) (Meißner et al. 2020) which takes into account whole complex of characteristics influencing the result image quality, including such variables as optical error of camera, atmospheric conditions etc. These approaches are based on visual analyses of different calibration targets, such as Bar target, Slanted edge test or Siemens star test to name few (Orych 2015).

Trends of spatial resolution related studies are described by Lee and Sung (Lee \& Sung 2016). Siemens star calibration target is one of the methods, which is repeatedly reported to be used, to evaluate GRD (Cramer 2013; Dabrowski \& Jenerowicz 2015; Dabrowski et al. 2015; Orych 2015). This test does not need any special equipment in terms of calibration target designing. Evaluation can be done based on visual analyses, without any special software. This target is also flexible in terms of flight direction and allows for defining resolution in a continuous way (Orych 2015).

This article is a first stage of study aimed at exploring the spatial resolution of images obtained by UAV DJI Phantom 3 Professional in different conditions. Following stage will explore how this spatial resolution influences the outcomes of typical Structure From Motion SFM processing pipeline resulting in surface point cloud of mixed forest stand.

Basic hypothesis assuming, that spatial resolution depends inter-alia on the flight altitude, flight speed and lighting conditions is examined in this first stage. The main aim of this work is to analyse which flight parameter, and with what impact is influencing the final GRD.

\section{Material and methods}

\subsection{Explanation of basic terms}

First of all, it is necessary to explain basic terms, which relates to the merit of our study. The terms are specifically Ground sampling distance, Ground resolved distance and Siemens star.
Ground sampling distance (GSD) in digital imagery represents the size of surface represented by single pixel of image. It is a theoretical measure that takes into account only geometry of camera (resolution of the sensor and lens focal distance) and distance to imaged surface or object. Other factors, such as system optics, interior noise, etc. are not considered (Orych 2015).

GSD can be calculated based on the real size of a single pixel on the sensor $x$, focal length of camera $f$ and distance between camera and target $h$ through formula:

$$
G S D=x * h / f
$$

Ground resolved distance (GRD) represents the smallest recognizable element on image. It can be determined by visual analysis on the basis of specific calibration targets, such as Siemens star (Orych 2015). Contrary to GSD, GRD is evaluated as a result of all factors influencing spatial resolution of imagery.

Siemens star represents the calibration target suitable for determination of GRD without any specific equipment other than target. Two types of Siemens star are used. Sinusoidal type for laboratory uses and Binary for outdoor testing. Binary Siemens star is formed by radial sectors alternating black and white color (Fig. 1a).

GRD determination using Siemens star with $\mathrm{n}$ sectors and diameter $\mathrm{D}$ is based on measuring the diameter $\mathrm{d}$ of blurred center up to the point where black and white sectors can be easily recognized (see Fig. $1 b$ ).

GRD can be then calculated based on formula:

$$
G R D=\pi * d / n
$$

GRD is key factor in subsequent analysis and data extraction from the imagery. And worse GRD definitely leads to lower success in studies dependent on pixel quality. For example, when detecting the tree position in small trees and regeneration of forest stands, or when trying to evaluate spectral reflectance on studies of tree health and physiology status (Klouček et al. 2019).

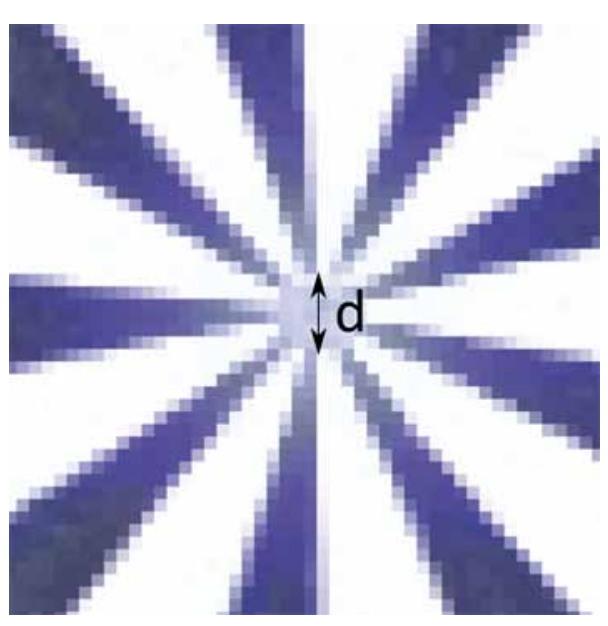

b)

Fig. 1. Siemens star calibration target used in study (a). Blurred center (with diameter d) of Siemens star calibration target (b). 


\subsection{Calibration target and study site}

Binary Siemens star with 18 sectors of $100 \mathrm{~cm}$ diameter (Fig. 1a) printed on paper and laminated to prevent damage, served as a calibration target. Such target in theory allows measuring GRD from 0 to $17.453 \mathrm{~cm}$. This should be sufficient, as the maximum planned flight height was $100 \mathrm{~m}$ with GSD $4.375 \mathrm{~cm}$.

Target was placed in a paved, level area allowing for undisturbed UAV flying in vicinity of Forest Management Institute in Brandýs nad Labem, Middle Bohemia region (WGS 84: 50.1876N, 14.6702E).

\subsection{Images acquisition}

DJI Phantom 3 Professional as an example of low-cost, user friendly, widely available UAV was used to acquire images. Parameters of camera DJI FC300X carried by this UAV are specified in Table 1 (DJI 2017).

Table 1. DJI FC300X Camera specification.

\begin{tabular}{ll}
\hline Sensor & Sony EXMOR 1/2.3" CMOS Effective pixels: $12.4 \mathrm{M}$ \\
\hline Lens & $20 \mathrm{~mm}(35 \mathrm{~mm}$ format equivalent) f/2.8 focus at $\infty$ \\
ISO Range & $100-1600$ (photo) \\
Electronic Shutter Speed & $8-1 / 8000 \mathrm{~s}$ \\
Image Size & $4000 \times 3000$ \\
\hline
\end{tabular}

Overall, four flight missions were performed to cover different light conditions, acquiring images in different altitudes roughly in 5-meter steps and different horizontal speed of flight. Camera pitch was set to 90 degrees and according to EXIF data originating from UAV Inertial Measurement Unit (IMU) this value was kept in all images with maximum deviance 0.1 degree, therefore all images are considered as nadir images.

\subsection{Images processing}

Non nadir exclusion: The distance between Siemens star target and image center is expected to influence GRD (Honkavaara et al. 2006a). As this study is primarily targeted on other influential conditions, images where Siemens star was out of the middle area of image defined according to grey area (see Fig. 2) were discarded, to eliminate influence of this variable.

EXIF metadata of relative altitude, $\mathrm{x}, \mathrm{y}, \mathrm{z}$ axis speed, aperture, exposure time and ISO, were extracted from all images using Exiftool utility (Harvey 2016). Images where $z$ axis speed was higher than $0.1 \mathrm{~m} / \mathrm{s}$ as well as images with ISO value other than 100 were discarded. Speed was calculated based on $\mathrm{x}, \mathrm{y}, \mathrm{z}$ axis speed vectors. Aperture was set to constant value (maximum aperture was used that equals to 2.0 in case of this camera) in camera settings prior to each flight. Still EXIF metadata were used to confirm (successfully), that all images are of the same aperture value.

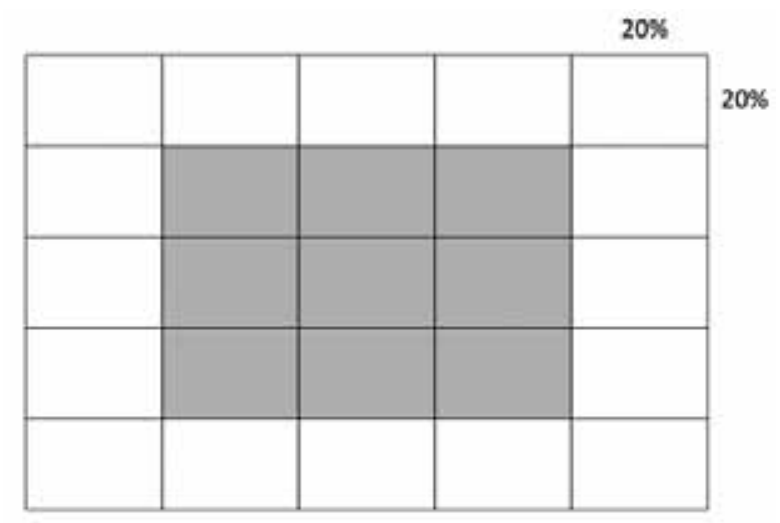

Fig. 2. Middle area (gray rectangles) of picture.

Using only images with same ISO and aperture allows to use exposure time as a descriptor of lighting conditions. Characteristics of resulting images set are summarized in Table 2.

\subsection{GRD determination}

In each image of resulting dataset, perimeter of blurred center area of Siemens star calibration target was measured using ImageJ software (Schneider et al. 2012).

Original RGB image was first transformed to 8-bit grayscale type using the ImageJ function Type/8bit which uses the formula gray $=($ red + green + blue)/3. In such image value of each pixel varies from 0 to 255 . Using threshold function, area with pixel values from 0 to certain value, well corresponding with blurred center area, was highlighted. In each of nine dark (highlighted) sectors the closest-to-the-center point was identified as a border of blurred center area.

As this area is rarely of regular circular shape, rather than measuring the diameter of this area, segmented line tool was used to connect all sectors and measure the perimeter $p$ of blurred area as in example Fig. 3a. Same area without applied threshold highlighting is in Fig. 3 b.

Table 2. Flight missions characteristics.

\begin{tabular}{llccccccc}
\hline \multirow{2}{*}{ Date } & \multirow{2}{*}{ Weather } & \multirow{2}{*}{ Images } & \multicolumn{2}{c}{$\begin{array}{c}\text { Altitude } \\
{[\mathrm{mAGL}}\end{array}$} & \multicolumn{2}{c}{$\begin{array}{c}\text { Speed } \\
{[\mathrm{m} / \mathrm{s}]}\end{array}$} & \multicolumn{2}{c}{$\begin{array}{c}\text { Exposure } \\
{[\mathrm{s}]}\end{array}$} \\
\cline { 3 - 9 } & & $\min$ & $\max$ & $\min$ & $\max$ & $\min$ & $\max$ \\
\hline $2019-07-26$ & Sunny, very light wind & 21 & 4.9 & 105.2 & 0.0 & 0.1 & $1 / 1750$ & $1 / 811$ \\
$2020-01-17$ & Sunny, very light wind & 60 & 30.3 & 100.8 & 0.0 & 14.4 & $1 / 514$ & $1 / 252$ \\
$2020-10-08$ & Partly cloudy, light wind & 64 & 6.2 & 70.3 & 0.0 & 12.6 & $1 / 736$ & $1 / 176$ \\
$2020-10-24$ & Mostly sunny, light wind & 39 & 10.0 & 70.0 & 0.0 & 9.5 & $1 / 561$ & $1 / 336$ \\
Total & 184 & 4.9 & 105.2 & 0.0 & 14.4 & $1 / 1750$ & $1 / 176$ \\
\hline
\end{tabular}




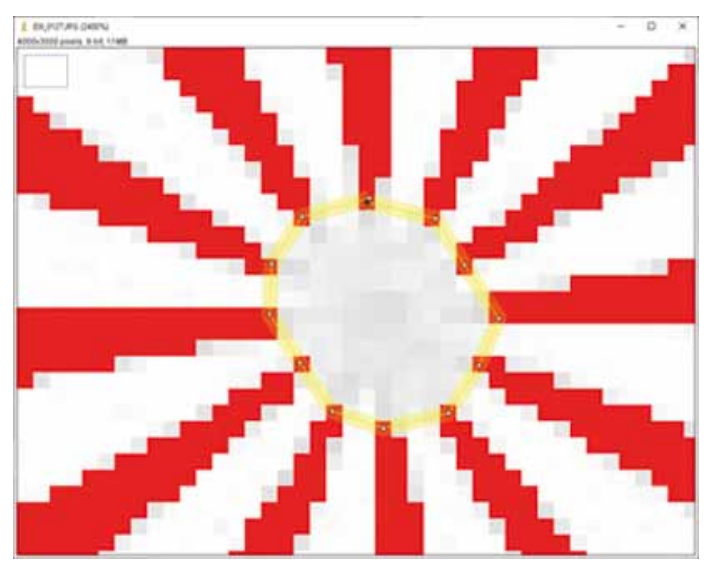

a)

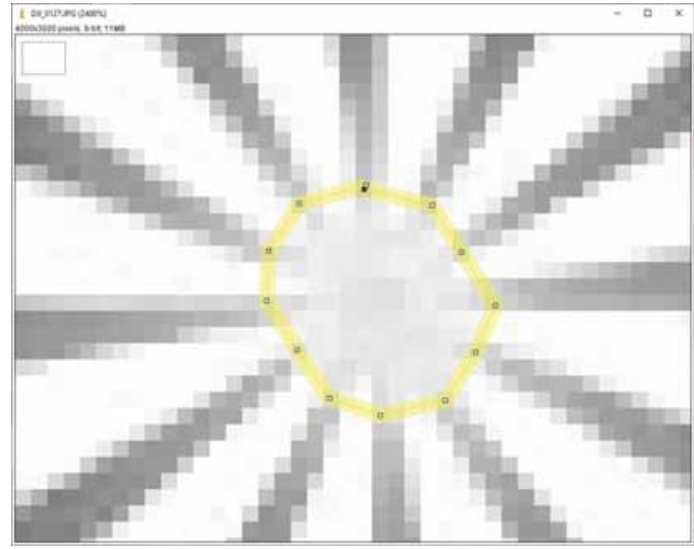

b)

Fig. 3. Identification of blurred center area with (a) and without threshold highlight (b).

Using this method, formula to determinate GRD can be adjusted accordingly with perimeter $p$ and number of segments $n$.

$$
\mathrm{GRD}=\mathrm{p} / \mathrm{n}
$$

GSD was derived from altitude and known parameters of camera according to formula 1 .

All statistical analyses were carried out in R-Studio version 1.4.1103 (RStudio Team 2019) with $R$ version 4.0.3 (R Core Team 2020) using following libraries: JTools(Long 2020), Readr (Wickham \& Hester 2020), GGplot2(Wickham 2016).

\section{Results}

In total 184 images from 4 flight missions were used for GRD evaluation (results overview is in Table 3).

Table 3. Basic statistics of GSD and GRD parameters of each of 184 images.

\begin{tabular}{|c|c|c|c|}
\hline & GSD & GRD & GRD/GSD \\
\hline & & & [unitless] \\
\hline$\overline{M i n}$ & 0.221 & 0.340 & 1.389 \\
\hline Median & 2.250 & 4.864 & 2.220 \\
\hline Mean & 2.335 & 5.205 & 2.189 \\
\hline Max & 4.734 & 12.113 & 3.087 \\
\hline
\end{tabular}

Impact of altitude on GRD is clearly visible in Fig. 4 with higher values of GRD in higher altitudes, though it is obvious that in higher altitudes additional factor influences the resulting GRD.

Inspecting the relationship between GRD and exposure time shows certain positive trend (Fig. 5). This can be translated as a better spatial resolution (lower GRD) is reached in better lighting conditions (shorter exposure time).

Also, relation between GRD and flight speed shows expected trend describing that with increasing speed the GRD is increasing (Fig. 6). In other words, spatial resolution gets worse with increasing speed.

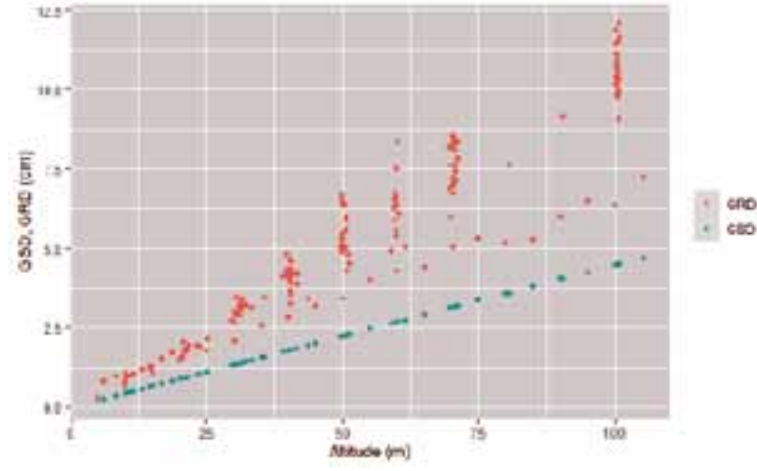

Fig. 4. Plot of GSD and GRD related to altitude.

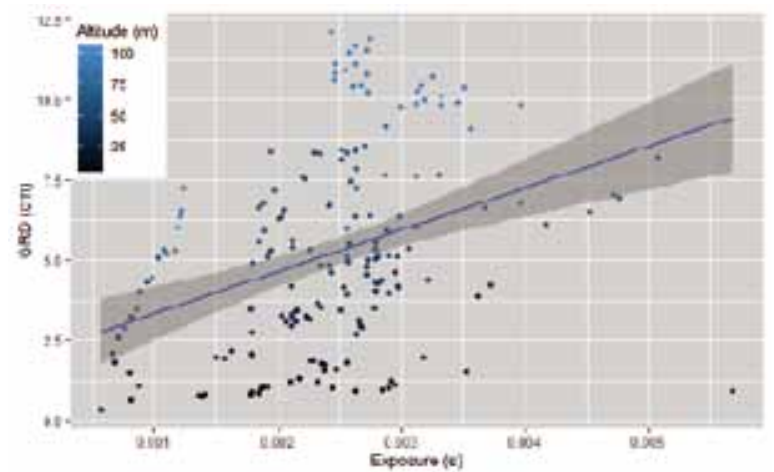

Fig. 5. Scatterplot of relation between GRD and exposure.

To describe GRD dependence on predictor variables (exposure, altitude and speed), linear regression methods were used.

First attempts led to models violating the basic linear model assumptions, namely the assumption of normally distributed residuals.

Therefore, different transformations of GRD variable were applied with the best results of square root transformation. This transformation used in stepwise modelling approach led to final linear model described in Table 4. 
Table 4. Results of linear model describing the relationship of square root of GRD as a dependent variable of predictors: Altitude, Speed and Exposure. $\mathrm{F}(3,180)=$ 855.306, $\mathrm{p}=<0.001, \mathrm{R}^{2}=0.934$, Adjusted $\mathrm{R}^{2}=0.933$. All predictors are mean-centered and scaled by 1 s.d.

\begin{tabular}{lcccc}
\hline Variable & Estimate & Std. Error & t value & p value \\
\hline (Intercept) & 2.167 & 0.014 & 159.527 & $<0.001$ \\
Altitude & 0.614 & 0.015 & 41.054 & $<0.001$ \\
Speed & 0.115 & 0.015 & 7.474 & $<0.001$ \\
Exposure & 0.066 & 0.014 & 4.561 & $<0.001$ \\
\hline
\end{tabular}

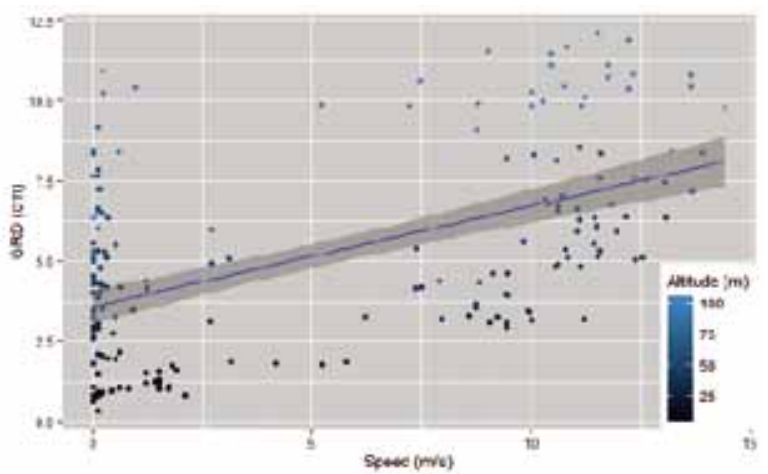

Fig. 6. Scatterplot of relation between GRD and speed.

Using Shapiro-Wilk normality test on residuals of this model resulted in $\mathrm{p}-$ value of 0.7285 , therefore normal distribution of residuals could not be denied. Linear model is described in Fig. 7, 8 and 9. Histogram of residuals and other results indicate good fit of the model.

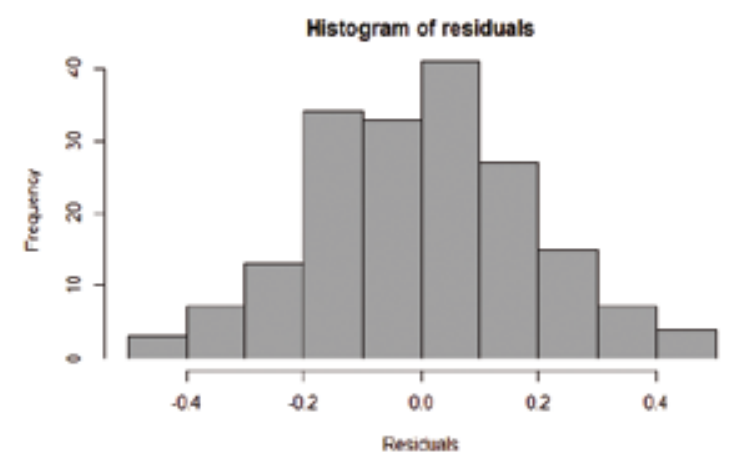

Fig. 7. Linear model - histogram of residuals.

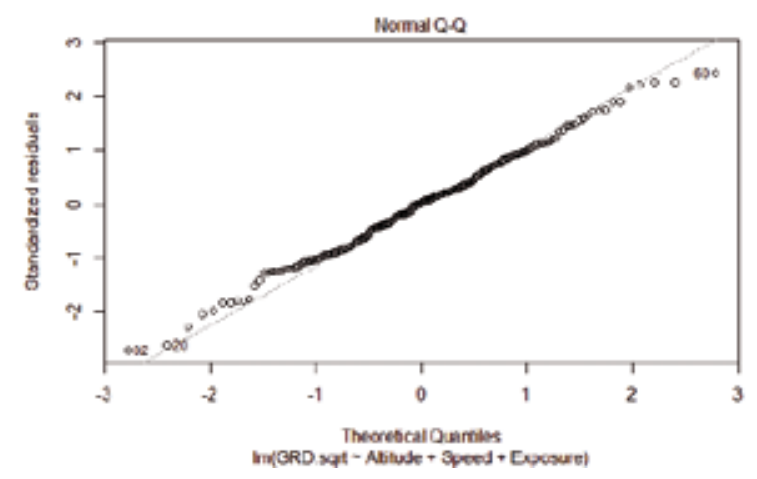

Fig. 8. Linear model-Q-Q plot.

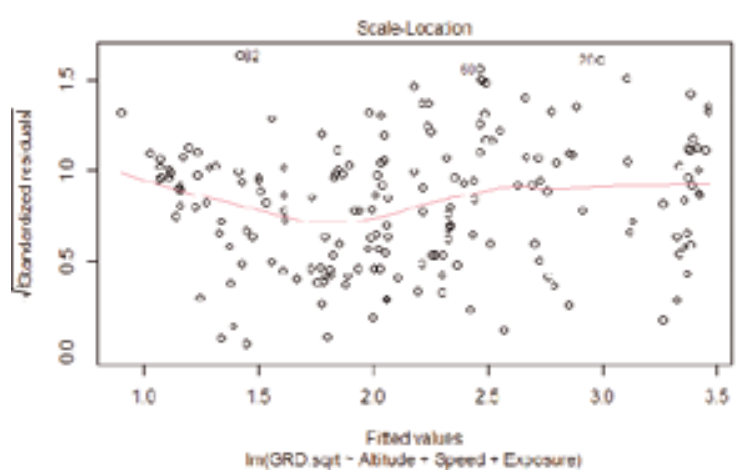

Fig. 9. Linear model - Scale - Location plot.

Further attempts on creating linear model with interactions between predictors led to only slightly better results in Adjusted $\mathrm{R}^{2}$ with high trade-offs in terms of increased complexity and lower explanatory value of the model.

\section{Discussion}

Recently mensuration of various variables by means of UAS become more and more available to broad audience, interested in the output. Though they don't need any more to study principles of UAV flying (propellers, flying mechanisms etc.) which are sufficiently handled by computers, they still face challenges how and when acquire the imagery to get the best results. Influence of different conditions (lighting conditions, flight height and flight speed) on resulting spatial resolution was examined in this study.

Existing studies targeted on UAV imagery acquiring mission options deal usually with GSD in terms of spatial resolution. This study goes further and demonstrates easy procedure to estimate more realistic spatial resolution in form of GRD for particular UAV setup, thus increasing the predictability of acquired image quality and also increasing the accuracy and precision of the results. Our main question was to provide readers with information: which of the selectable flight parameters height, speed, light has the highest influence on final quality and which is lowest (potentially could be sacrificed in favor of the most important one).

Evaluation of presented linear model, apart from expected negative influence of altitude on spatial resolution, proved also negative influence of speed and positive influence of better lighting conditions. In other, more general words, results of this study confirm, that best spatial resolution of UAV imagery can be reached by performing flight missions in lower flight height, with lower flight speed and better conditions in terms of available light. These results are in line with expected outcomes, although the influence of lighting conditions seems to be rather minor. 
Results show, that GRD was always higher than GSD as GRD/GSD ratio takes on values from 1.4 to 3.1 with mean value 2.2. Similar trend with higher GRD compared to GSD was published by Lee (Lee \& Sung 2016) although the difference was much lower with GRD/GSD ratio between 1.2 and 1.4. Cameras used in quoted study (Lee \& Sung 2016) were Canon (IXUS 127 HS) and Sony (NEX-5 N), both of them of better specification than camera used in this study.

Similar results were achieved by Dabrowski (Dabrowski et al. 2015). In this case GRD was examined as a linear function of altitude with GRD/GSD ratio approximately 2.6 and 2.5 for Sony (NEX-5) and miniMCA camera respectively.

GRD to GSD ratio of $1.3,1.8$ and 1.3 was reported in (Cramer \& Zhang 2020), using images of Siemens star captured by DJI Phantom 4, DJI Phantom 4 RTK and Phase One iXM100-RS camera in flight altitudes 28,25 and 47 respectively.

Resolving power derived by the means of Siemens star target on imagery obtained by Intergraph DMC digital large-format photogrammetric sensor carried on airplane led to 1.2 to 1.5 times higher results than GSD as concluded Honkavaara (Honkavaara et al. 2006a, b).

Worse spatial resolution was observed on images taken at higher horizontal flight speed. These results can be compared to much higher GRD to GSD ratio (10 to 15 times higher) of images obtained by S.O.D.A camera on SenseFly eBee Plus fixed wing UAV in (Stöcker et al. 2018). Quoted study does not state the values of UAV horizontal flying speed, but considering the fixed wing design of UAV in this case, higher speed might be one of the reasons for worse results of spatial resolution, same way as our study suggests. Results also indicate positive relation of spatial resolution to better lighting conditions. This is in line with Lim (Lim et al. 2018a), who reported different results of GRD based on lighting conditions. Using Sony A5100 camera carried on octocopter UAV, GRD was 1.1 to 2.3 (depending on the flight altitude) times higher than GSD in images taken on sunny conditions, whereas 1.6 to 3.7 times higher than GSD in images obtained at cloudy day.

Similar relationship to lighting conditions were reported in (Lim et al. 2018b). Images of Siemens star, captured by octocopter carried Sony A5100 in altitudes 20 to 80 meters, resulted in GRD 3-12 times higher than GSD in very cloudy day comparing to 1.2 to 3 times higher GRD than GSD in sunny or little cloudy day.

\section{Conclusions}

Our work focused on description of the first stage of exploring processes for the optimal ways to quantify characteristics of remote sensing data obtained by common commercial UAV (in our case DJI Phantom 3 Professional UAV) in forestry. The main motivation was to provide the reader and potential practitioner of forest mensuration by the means of remote sensed data with information about the impact of different height, speed and possible light condition on the image quality. In general, the image quality is clearly linked to quality and accuracy of information obtained from such image by means of photogrammetry.

Spatial resolution of imagery is considered as one of the most important preconditions influential to any result of remote sensing application. Therefore, special care was given in this first stage to Ground resolved distance as an objective means to describe spatial resolution. Influence of different conditions in form of flight altitude, flight speed and lighting conditions on resulting spatial resolution was examined. Study proved, that spatial resolution is highly dependent on flight altitude with better resolution resulting from lower flight altitudes, less influential, but still highly significant proved flight speed. Comparing to previous two factors, lighting conditions, although still significant, are of the least influence. This can be translated as a positive message for the flexibility of use of UAV in forestry, as the most unpredictable characteristics of flight mission, lighting conditions as a result of the weather conditions, seems to be of rather low impact on resulting spatial resolution. Concluding knowledge both from previous studies and this work, UAVs can be recommended for forestry sector as a reliable device in terms of imagery data acquisition in forest stands and open areas.

\section{Acknowledgments}

This research was funded by Faculty of Forestry and Wood Sciences Prague. The authors would like to thank Radim Adolt (Forest Management Institute, Brandýs nad Labem) for his support in performing the statistical analyses.

\section{References}

Cramer, M., 2013: The UAV@LGL BW Project - A NMCA Case Study. In: Fritsch, D. (ed.): Photogrammetric Week 2013. Wichmann/VDE Verlag, Belin \& Offenbach, p. 165-179.

Cramer, M. , Zhang, S., 2020: Quality Assessment of High-Resolution UAV Imagery and Products. In: 40. Wissenschaftlich-Technische Jahrestagung der DGPF in Stuttgart, p. 33-46.

Dabrowski, R. , Jenerowicz, A., 2015: Portable Imagery Quality Assessment Test Field for UAV Sensors. In: The International Archives of the Photogrammetry, Remote Sensing and Spatial Information Sciences ISPRS Archives, 40:117-122. 
Dabrowski, R., Orych, A., Jenerowicz, A., Walczykowski, P., 2015: Preliminary Results from the Portable Imagery Quality Assessment Test Field (PIQuAT) of UAV Imagery for Imagery Reconnaissance Purposes. In: The International Archives of the Photogrammetry, Remote Sensing and Spatial Information Sciences - ISPRS Archives, 40:111-115.

Harvey, P., 2016: ExifTool. Available in: http://exiftool. org.

Honkavaara, E., Jaakkola, J., Markelin, L., Becker, S., 2006a: Evaluation of Resolving Power and MTF of DMC. In: The International Archives of the Photogrammetry, Remote Sensing and Spatial Information Sciences - ISPRS Archives, 36:1-6.

Honkavaara, E., Jaakkola, J., Markelin, L., Peltoniemi, J., Ahokas, E., Becker, S., 2006b: Complete Photogrammetric System Calibration and Evaluation in the Sjökulla Test Field - Case Study With Dmc Abstract. In: Proceedings of EuroSDR Commission I and ISPRS Working Group 1/3 Workshop EuroCOW 2006, 6 p.

Klouček, T., Komárek, J., Surový, P., Hrach, K., Janata, P., Vašíček, B., 2019: The Use of UAV Mounted Sensors for Precise Detection of Bark Beetle Infestation. Remote Sensing, 11:1-17.

Lee, J., Sung, S., 2016: Evaluating Spatial Resolution for Quality Assurance of UAV Images. Spatial Information Research, 24:141-154.

Lim, P. C., Kim, T., Na, S. Il, Lee, K. Do, Ahn, H. Y., et al., 2018a: Analysis of Uav Image Quality Using Edge Analysis. In: The International Archives of the Photogrammetry, Remote Sensing and Spatial Information Sciences - ISPRS Archives, 42:425-32.

Lim, P. C., Seo, J., Kim, T., 2018b: Extraction of UAV Image Sharpness Index Using Edge Target Analysis. Korean Journal of Remote Sensing, 34:905-23.

Long, J. A., 2020: Jtools: Analysis and Presentation of Social Scientific Data. Available in: https://cran.rproject.org/package $=$ jtools
Meißner, H., Cramer, M., Reulke, R., 2018: Towards Standardized Evaluation of Image Quality for Airborne Camera Systems. In: The International Archives of the Photogrammetry, Remote Sensing and Spatial Information Sciences - ISPRS Archives, 42:295-300.

Meißner, H., Cramer, M., Reulke, R., 2020: Evaluation of Structures and Methods for Resolution Determination of Remote Sensing Sensors. Lecture Notes in Computer Science (Including Subseries Lecture Notes in Artificial Intelligence and Lecture Notes in Bioinformatics), 11994 LNCS: 59-69.

Orych, A., 2015: Review of Methods for Determining the Spatial Resolution of UAV Sensors. In: The International Archives of the Photogrammetry, Remote Sensing and Spatial Information Sciences - ISPRS Archives. 40:391-395.

Pricope, N. G., Mapes, K. L., Woodward, K. D., Olsen, S. F., Britton Baxley, J., 2019: Multi-Sensor Assessment of the Effects of Varying Processing Parameters on Uas Product Accuracy and Quality. Drones, 3:1-17.

R Core Team 2020: R: A Language and Environment for Statistical Computing. Available in: https://www.rproject.org/

RStudio Team 2019: RStudio: Integrated Development Environment for R. Available in: https://www.rstudio.com/

Schneider, C. A., Rasband, W. S., Eliceiri, K. W., 2012: NIH Image to ImageJ: 25 Years of Image Analysis. Nature Methods, 9:671-675.

Stöcker, C., Koeva, M., Nex, F., 2018: Deliverable 4.2 Data Quality Assessment of UAV-Based Products for Land Tenure Recording. H2020 its4land 687828, $55 \mathrm{p}$.

Wickham, H., 2016: Ggplot2: Elegant Graphics for Data Analysis. Springer-Verlag New York, 213 p.

Wickham, H., Hester, J., 2020: Readr: Read Rectangular Text Data. Available in: https://CRAN.R-project. org/package $=$ readr 